\title{
Erratum: Carrier recombination mechanism at defects in wide band gap two-dimensional materials from first principles [Phys. Rev. B 100, 081407(R) (2019)]
}

\author{
Feng Wu, Tyler J. Smart, Junqing Xu, and Yuan Ping •
}

(Received 15 June 2021; published 4 August 2021)

DOI: 10.1103/PhysRevB.104.079901

Two issues need to be addressed in the original paper:

(1) Nonradiative recombination rates of defect-defect transitions [recombination between two localized defect states in the hexagonal boron nitride ( $h$-BN) band gap] should converge with respect to supercell sizes to represent the dilute limit where defects do not interact. This is different from the nonradiative transitions between defect and bulk states (e.g., band edges of $h$-BN) where the rates depend on the defect concentration explicitly, and a capture coefficient is defined accordingly to remove defect concentration dependence. Therefore, we need to remove the capture coefficient definition in Tables II, S1, and S4 in the original paper. And the supercell convergence in Table S1 needs to be reexamined and has been corrected. Figure E1 and Table EI are new in order to provide more technical details.

(2) Similarly, radiative recombination of defect-defect transitions at a dilute limit should be evaluated without momentum dependence of excitons (no interaction among electron-hole pairs from neighboring defects), similar to zero-dimensional systems embedded in a dielectric medium [1-3]. For ultrathin two-dimensional (2D) materials where both the in-plane and out-of-plane dielectric constants reduce to one, the radiative rate is given by

$$
\Gamma_{R}=\frac{e^{2}}{3 \pi \epsilon_{0} \hbar^{4} c^{3}} E_{0}^{3} \mu_{e-h}^{2}
$$

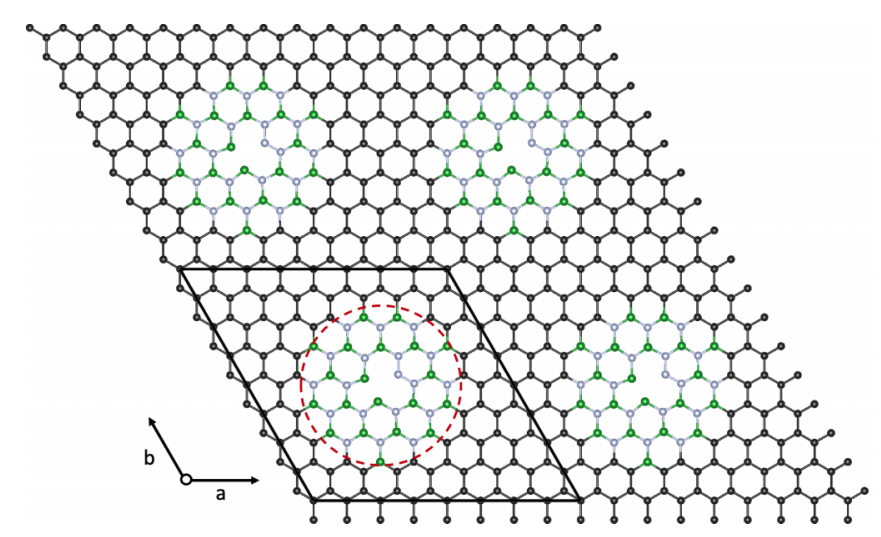

FIG. E1. Atomic structure of $N_{\mathrm{B}} V_{\mathrm{N}}$ in monolayer $h$-BN with a $8 \times 8$ supercell (black box). Boron (green) and nitrogen (gray) atoms in color are within the radius of atoms which are fully relaxed (red dashed circle with a radius of $6 \AA$ ), and atoms outside of this circle are fixed (black) to their pristine atomic positions. This is a new figure, not in the original paper. 

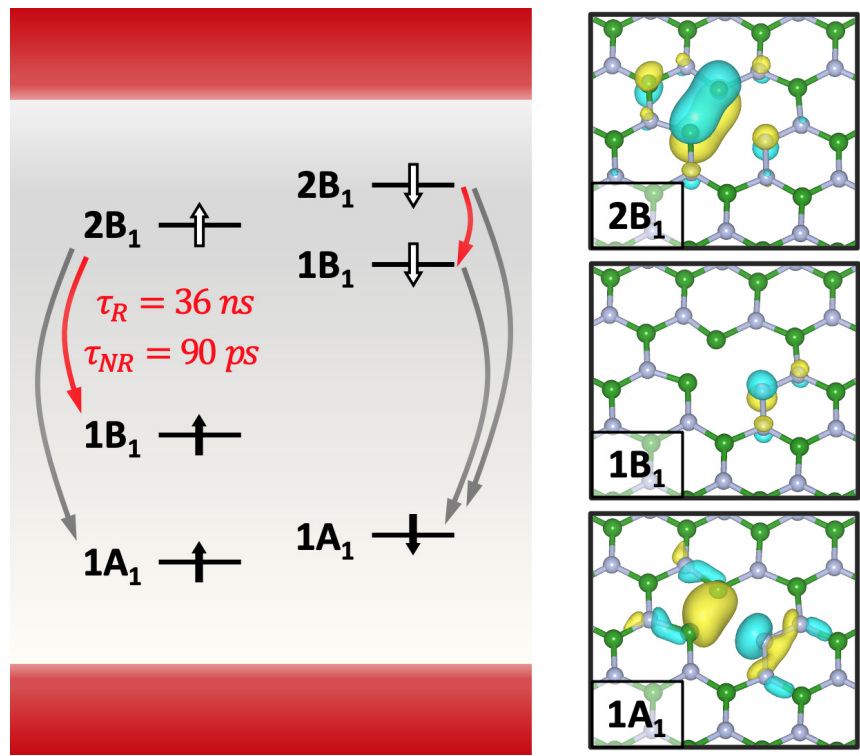

FIG. 2. Defect levels and possible defect-defect transitions of $N_{B} V_{N}$ in monolayer $h$-BN. Both up and down spin channels of the $2 \mathrm{~B}_{1} / 1 \mathrm{~B}_{1}$ transitions are marked in red as they are optically allowed with light polarized along defect $C_{2}$ symmetry axis.

TABLE S1. Nonradiative recombination lifetime (at $T=300 \mathrm{~K}$ ) of $N_{\mathrm{B}} V_{\mathrm{N}}$ transition $1 B_{1} \uparrow / 2 B_{1} \uparrow$ at the PBE as a function of supercell sizes. Fixed-atom calculations use a radius of $6 \AA$ as shown in Fig. E1.

\begin{tabular}{|c|c|c|c|c|c|c|}
\hline Cell & \multicolumn{3}{|c|}{ Free relaxation } & \multicolumn{3}{|c|}{ Fixed atoms } \\
\hline $6 \times 6$ & 0.15 & 1.26 & 102 & 0.42 & 4.38 & 24 \\
\hline $8 \times 8$ & 0.13 & 1.06 & 135 & 0.37 & 1.50 & 90 \\
\hline $10 \times 10$ & 0.11 & 0.45 & 358 & 0.39 & 0.83 & 146 \\
\hline
\end{tabular}

TABLE S2. Radiative recombination lifetime of $N_{\mathrm{B}} V_{\mathrm{N}}$ transition $1 B_{1} \uparrow / 2 B_{1} \uparrow$ computed by DFT-RPA as a function of supercell sizes. The $\Gamma$ point is used for the Brillouin-zone sampling for a balance of computational cost and consistency between different supercell sizes.

\begin{tabular}{lccc}
\hline \hline Cell & $|\mu|^{2}($ a.u. $)$ & $E_{\text {ex }}(\mathrm{eV})$ & $\tau^{R}(\mathrm{~ns})$ \\
\hline $6 \times 6$ & 1.37 & 2.45 & 47 \\
$8 \times 8$ & 1.48 & 2.48 & 42 \\
$10 \times 10$ & 1.57 & 2.48 & 39 \\
$12 \times 12$ & 1.36 & 2.48 & 45 \\
\hline \hline
\end{tabular}

TABLE EI. Non-radiative recombination lifetime (at $T=300 \mathrm{~K}$ ) of $N_{\mathrm{B}} V_{\mathrm{N}}$ transition $1 B_{1} \uparrow / 2 B_{1} \uparrow$ at Perdew-Burke-Ernzerhof (PBE) with a $10 \times 10$ supercell as a function of the fixing-atom radius. This is a new table, not in the original paper.

\begin{tabular}{lcccccc}
\hline \hline Radius $(\AA)$ & $\begin{array}{c}\text { Zero-phonon line energy } \\
(\mathrm{eV})\end{array}$ & $\begin{array}{c}\Delta Q \\
(\sqrt{\mathrm{amu}}) \AA\end{array}$ & $\begin{array}{c}\hbar \omega_{f} \\
(\mathrm{eV})\end{array}$ & $S_{f}$ & $\begin{array}{c}\left|W_{i f}\right|^{2} \\
\left(\mathrm{eV}^{2} \mathrm{amu} \AA^{2}\right)\end{array}$ & $\begin{array}{c}X_{i f} \\
(\mathrm{a} . \mathrm{u} .)\end{array}$ \\
\hline 5 & 2.24 & 0.586 & 113.1 & 4.64 & 0.39 & 0.41 \\
6 & 2.18 & 0.611 & 109.71 & 4.89 & 0.39 & 0.83 \\
7 & 2.15 & 0.616 & 108.49 & 4.92 & 0.39 & 0.94 \\
8 & 2.15 & 0.606 & 110.17 & 4.83 & 0.40 & 1.10 \\
\hline \hline
\end{tabular}


TABLE S3. Radiative lifetime of $2 B_{1} \uparrow / 1 B_{1} \uparrow$ computed by DFT-RPA and $G W$-BSE for the $N_{\mathrm{B}} V_{\mathrm{N}}$ defect with a $6 \times 6$ supercell and $3 \times 3$ $k$-point meshes.

\begin{tabular}{lccc}
\hline \hline Method & $|\mu|^{2}$ (a.u.) & $E_{e x}(\mathrm{eV})$ & $\tau^{R}(\mathrm{~ns})$ \\
\hline DFT-RPA & 1.53 & 2.34 & 48 \\
$G W$-BSE & 1.87 & 2.41 & 36 \\
\hline \hline
\end{tabular}

where $e$ is the charge of an electron, $\epsilon_{0}$ is vacuum permittivity, $E_{0}$ is the exciton energy at the long-wavelength limit $\mathbf{Q}_{e x}=0$ and $\mu_{e-h}^{2}$ is the modulus square of exciton dipole moment with length ${ }^{2}$ unit. This issue impacts Fig. 2, Table S2, and Table S3 in the original paper where we should remove the capture coefficient definition and use Eq. (1) for isolated systems instead of the rate for $2 \mathrm{D}$ systems. Therefore, here we replaced them by the corrected versions.

To address the first issue, we perform supercell convergence tests again for nonradiative rates of defect-defect transitions. The supercell convergence was very slow in our original paper due to the slow decay of strain in the bulk region induced by the point defect; therefore, we use the method of fixing atoms away from the defect region wherein forces at the boundary are zero, analogous to the approach used in Ref. [4]. As such, we test the nonradiative rates as a function of the radius outside of which the atoms are fixed as shown in Fig. E1. Once we determined an appropriate radius, we then retest supercell convergence for nonradiative rates. For the second issue, we simply report the radiative lifetime for defect-defect transition at the dilute limit using Eq. (1). We note that the majority of our conclusions from the original publication still hold. More details are as follows.

With the same supercell size $($ e.g., $10 \times 10)$, the nonradiative rates are found to have small changes with fixed atoms beyond the radius of $6 \AA$ as shown in Table EI and Fig. E1. Next nonradiative recombination rates for various supercell sizes are shown in the case of free relaxation (left side, original Table S1) and with fixed atoms beyond the radius of $6 \AA$ (right side) in corrected Table EII . Unlike the case of free relaxation with fixed atoms, nonradiative lifetime converges at an $8 \times 8$ supercell to be on the order of $\sim 100 \mathrm{ps}$. The error bar on the nonradiative lifetime is $\sim \pm 30 \%$, which is vastly improved but still not ideal. This is a known issue which requires new method development. Note this value is, by some coincidence, very similar to the original reported nonradiative lifetime given the error bar above (102 ps, obtained by a $6 \times 6$ supercell with free relaxation).

For the radiative lifetime, we previously reported values using formula for $2 \mathrm{D}$ systems, which consider interaction among periodic images of defects that lead to exciton dispersion in two-dimensional momentum space (values reported in Fig. 2, Table S2, and Table S3 for the $2 B_{1} \uparrow / 1 B_{1} \uparrow$ transition also in the text radiative lifetime reported for the $2 B_{1} \downarrow / 1 B_{1} \downarrow$ transition in the original paper). As aforementioned at the dilute limit we use Eq. (1) for defect-defect radiative recombination with the same inputs of transition dipole moment and excitation energies as were previously used. For the $2 B_{1} \uparrow / 1 B_{1} \uparrow$ transition, the supercell convergence of radiative lifetime using random phase approximation (RPA) starting from density functional theory (DFT) single-particle (DFT-RPA) states is shown in corrected Table S2, wherein a $6 \times 6$ supercell is sufficient to converge the radiative lifetime to be on the order of $\sim 40 \mathrm{~ns}$, even for fully relaxed structures (a value of $1.4 \mathrm{ps}$ was reported in the original paper). For the $2 B_{1} \downarrow / 1 B_{1} \downarrow$ transition we find a radiative lifetime of $250 \mathrm{~ns}$ (a value of $2.5 \mathrm{ps}$ was reported in the original paper). In order to include accurate electron correlation and electron-hole interactions, many-body perturbation theory $G W$-BSE calculations are shown in corrected Table S3 with a radiative lifetime of $36 \mathrm{~ns}$. More details of $G W$-BSE calculations can be found in the original paper. In final, we provide updated values for Fig. 2, Table S2, and Table S3. Although discussions related to defect-defect nonradiative lifetime in Table II in the original paper remain unchanged, the updated value for the radiative lifetime leads to a different interpretation of Fig. 2. Namely, $N_{\mathrm{B}} V_{\mathrm{N}}$ is found to have a radiative lifetime which is three orders of magnitude longer than its nonradiative transition and, therefore, it does not possess a high quantum yield $(0.25 \%)$. This is a consequence of its strong electron-phonon coupling (e.g., large Huang-Rhys factor) which leads to a dominant phonon-assisted nonradiative recombination.

The last note is that for the defect-band transition (recombination between localized defect states and band edges of $h$-BN) in Table I of the original paper, the capture coefficients with nonradiative lifetimes longer than milliseconds can be considered as zero (originally they were given on the order of $10^{-30} \mathrm{~cm}^{2} / \mathrm{s}$ ). This is because with such small capture coefficients, it is sensitive to numerical parameters, such as smearings used in the Gaussian functions for energy conservation. Implementation similar to Ref. [5] may provide numerically more accurate results; nevertheless, these processes are extremely slow, thus, less important.

We acknowledge A. Alkauskas for helpful discussions. This work was supported by the National Science Foundation under Grant No. DMR-1760260. This research used resources of the Center for Functional Nanomaterials, which is a US DOE Office of Science Facility, and the Scientific Data and Computing Center, a component of the Computational Science Initiative, at Brookhaven National Laboratory under Contract No. DE-SC0012704, and the lux supercomputer at UC Santa Cruz, funded by National Science Foundation MRI Grant No. AST 1828315.

[1] F. Wu, D. Rocca, and Y. Ping, J. Mater. Chem. C 7, 12891 (2019).
[2] M. Mackoit-Sinkeviāienè, M. Maciaszek, C. G. Van de Walle, and A. Alkauskas, Appl. Phys. Lett. 115, 212101 (2019). 
[3] T. J. Smart, K. Li, J. Xu, and Y. Ping, npj Comput. Mater. 7, 59 (2021).

[4] A. Alkauskas, B. B. Buckley, D. D. Awschalom, and C. G. Van de Walle, New J. Phys. 16, 073026 (2014).
[5] M. E. Turiansky, A. Alkauskas, M. Engel, G. Kresse, D. Wickramaratne, J.-X. Shen, C. E. Dreyer, and C. G. Van de Walle, Comput. Phys. Commun. 267, 108056 (2021). 